Egyptian Poultry Science Journal

http://www.epsaegypt.com

ISSN: 1110-5623 (Print) - 2090-0570 (Online)

\title{
IMPACT OF STRAIN, EGG TYPE, BREEDER AGE AND THEIR INTERACTIONS ON HATCHING TRAITS AND CHICK QUALITY
}

\section{G. N. Rayan}

Dep. of Poult. Prod., Fac. of Agric., Ain Shams Uni., Cairo, Egypt.

Corresponding author: Gamal_Rayan@agr.asu.edu.eg

\begin{abstract}
Received: $24 / 01 / 2018$
Accepted: $12 / 02 / 2018$

ABSTRACT: Eight hundred hatching eggs from two Hy-Line chicken strains (400 Brown and $400 \mathrm{~W}-36)$ were acquired from two layer breeder ages (46 and 60 weeks). In each age there are four egg types (normal, large, rough and shape abnormality), each type containing fifty hatching eggs from each age either for Brown or W-36 chicken strains. Thus, this experiment was a $2 \times 2 \times 4$ factorial design. There were no significant differences between strains for relative egg weight loss. Egg weight loss (\%) increases with the breeder age. Relative egg weight loss was significantly higher in rough and abnormal eggs compared to their counterparts. Rough eggs type of brown strain at different breeder age had the lowest fertility and hatchability percentages compared to other egg types. Abnormal shape egg type had the lowest value of shape index. Brown chickens produced eggs had significantly higher relative eggshell weight compared to the white one. Shell percentage significantly decreases with advancing of hen's age. White eggs significantly recorded higher total pores per egg (7583) compared to the brown one (7249). Total pores significantly increases with breeder age. Large egg type was significantly higher in total pores compared to other egg types. Eggs with rough shell type had the highest embryonic mortality percentage in early stage (0-7 d of incubation) for each brown and W-36 strains at different ages, followed by eggs with shape abnormality compared to other egg types. Large egg type was significantly higher in hatched chick weight $(46.85 \mathrm{~g})$, then shape abnormality (41.12 g). There were no significant differences between strains for Tona score as indication of chick quality. Tona score decreased as the layer breeder age progressed. Rough egg type had the lowest value of Tona score $(78.25 \%)$; whereas, no significant difference was observed between other studied egg types (normal; 99.80, large; 95.65, and abnormal; $95.25 \%$ ). Tona score was significantly affected by all potential interactions, that means the expression of this trait was different based on strain, layer breeder age and egg type. It could be concluded that practically we can benefit from large and shape abnormality eggs, because there is no significant difference between them and normal eggs for most eggshell characteristics, chick quality and fertility \& hatchability percentages.
\end{abstract}

Keywords: Strain - Egg type - Breeder age - Chick quality - Hatching traits. 


\section{INTRODUCTION}

In hatcheries, the main target is to maximize hatchability with a lot of high chick quality, saleable chicks that are demand by producers for their high performance and viability (Decuypere and Bruggeman, 2007). Study of factors that effect the production of high quality chicks is of great attention to hatching egg producers because they are paid based on the number of salable chicks produced (not hatched). Quality of hatching eggs is very important because eggs provide both physical protection and nutrition for the growing embryo. Shell quality (pores number and shell thickness) determines gas exchange and humidity loss during incubation. Poor eggshell quality has been related with a higher percentage of egg moisture loss during incubation (Peebles et al., 2001), low hatchability (Narushin and Romanov, 2002). Also, breeder age may effect on hatching quality. Lourens et al. (2006) reported that chicks hatched from large eggs have higher body weight also develop faster because of the higher availability of nutrients existing in the egg compared with those from small eggs. Hamidu et al. (2007) reported that young breeders lay eggs with thicker eggshell than those of older breeders, and therefore, less oxygen is supplied to the embryos, which may clarify their slower metabolism and development. Some reasons for low hatchability could be due to unsuitable management of the breeder flock, or failure within any step between breeder flock and final hatch i.e embryonic mortality. Therefore, optimizing of hatchery and breeder management can lead to improve chick's production (Heier and Jarp, 2001). Factors concerning breeder that affect hatchability include strain, breeder age, size of egg, egg quality and duration of egg storage (Wilson, 1997; Tona et al., 2005). Chick quality at hatch dependent on many factors including age of breeder, strain, quality of hatching eggs, chick health, egg handling and storage (Peebles et al., 2001; Tona et al., 2003; Decuypere and Bruggeman, 2007). Tona et al. (2001) reported that age of breeders (layer and broiler) is an important to be considered by the hatchery managers. Also, they stated that eggs produced from young or older breeder don't hatch like the eggs from the breeders of 40 to 42 weeks of age. Therefore, the main objective of this research was to study the impacts of strain, egg type, breeder age and their interactions on hatching traits and chick quality.

\section{MATERIALS AND METHODS}

This experiment was carried out at the hatchery of EL-Mansouria Poultry Misr Company situated at EL-Mansouria area October Governorate. Eight hundred hatching eggs from two Hy-Line strains (400 Brown and $400 \mathrm{~W}-36$ ) were acquired from two layer breeder ages (46 and 60 weeks). Eggs were stored for 3-7 days at $15^{\circ} \mathrm{C}$ and $80 \%$ relative humidity until incubation. After delivering the eggs to the hatchery, eggs were checked and sorted. In each age there are four egg types (normal, large, rough and shape abnormality), each type containing fifty hatching eggs from each age either for Brown and $\mathrm{W}-36$. Thus, this experiment was a $2 \times 2 \times 4$ factorial design, with 2 strains, 2 ages and 4 egg types. Eggs incubated in Petersime 


\section{Strain - Egg type - Breeder age - Chick quality - Hatching traits.}

incubator at $37^{\circ} \mathrm{C}$ and $65 \%$ relative humidity.

\section{Egg weight \& Egg weight loss}

Before incubation, eggs from each type from the two ages within each chicken strain were individually weighed to the nearest $0.01 \mathrm{~g}$ using an electronic digital balance, before setting in the incubator and again at $18^{\text {th }}$ day of incubation to calculate egg weight loss. Percentage of egg weight loss during incubation period (0-18 days) was calculated as follow:

Egg weight loss $(\%)=$ Egg wt. at the beginning of incubation - Egg wt. at 18 days of incubation / Egg wt. at the beginning of incubation $\mathrm{x} 100$

Fertility \& Hatchability (\%)

Eggs were candled at $7^{\text {th }}$ day of incubation. Fertility (\%) was calculated by dividing number of fertile eggs on number of settable eggs $\mathrm{x} 100$. While, hatchability (\%) was calculated by dividing number of hatched chicks on number of fertile eggs $\mathrm{x}$ 100.

\section{Egg and eggshell traits}

Egg length and width were individually recorded by a digital caliper. Then, shape index $=$ (width $/$ length $) \times 100$.

Wet shell weight (to the nearest $0.01 \mathrm{~g}$ ) was measured after hatching.

Shell percentage $=$ (wet eggshell weight $/$ egg weight) $\mathrm{x} 100$.

Number of pores in eggshell was calculated depending on Rahn and Paganelli (1990) equation:

Pores number $(\mathrm{N})=304 \mathrm{M}^{0.767}$

Where: $\mathrm{M}=$ Egg weight.

Eggshell surface area (SA) was calculated using the following equation according to Fathi and El-Sahar (1996):
SA $=9.07$ (Egg length $x$ Breadth $)^{0.63}$

Shell index $\left(\mathrm{g} / 100 \mathrm{~cm}^{2}\right)$ was calculated depending on equation of Sauveur (1988):

$\mathrm{SI}=$ [Shell weight $/$ shell surface area $] \mathrm{x}$ 100

\section{Stages of embryonic mortality}

After hatching, all unhatched eggs were opened to determine embryonic mortality stage as can as possible: early ( 1 to $7 \mathrm{~d}$ ), mid ( 8 to $14 \mathrm{~d}$ ) and late ( 15 to $21 \mathrm{~d}$ ).

\section{Chick quality}

After hatching, all chicks were removed at 21.5 days of incubation and the chick quality tests were determined to measure the quantitative and qualitative score factors of chicks.

One-day chicks were individually weighed. Chicks were examined to identify the various traits that can be related to good, medium or poor quality chicks depending on varying physical appearances of day-old chicks. Chick quality was determined according to Tona et al. (2003).

\section{Statistical analysis:}

Data were analyzed using three-way analysis of variance for strain, layer breeder age, egg type and their interaction using the General Linear Model (GLM) procedure of SAS (2002) as following model;

$\mathrm{Y}_{\mathrm{ijk} l}=\mu+\mathrm{S}_{\mathrm{i}}+\mathrm{A}_{\mathrm{j}}+\mathrm{T}_{\mathrm{k}}+\left(\mathrm{S}^{*} \mathrm{~A}\right)_{\mathrm{ij}}+\left(\mathrm{S}^{*} \mathrm{~T}\right)_{\mathrm{ik}}+$ $\left(\mathrm{A}^{*} \mathrm{~T}\right)_{\mathrm{jk}}+\left(\mathrm{S}^{*} \mathrm{~A}^{*} \mathrm{~T}\right)_{\mathrm{ijk}}+\mathrm{e}_{\mathrm{ijkl}}$

Where;

$\mathrm{Y}_{\mathrm{ijkl}}=$ Trait measured,

$\mu=$ Overall means,

$\mathrm{S}_{\mathrm{i}}=$ Strain effect $(\mathrm{i}=1,2)$,

$A_{j}=$ layer breeder age effect $(j=1,2)$,

$\mathrm{T}_{\mathrm{k}}=$ Egg type effect $(\mathrm{k}=1,2,3,4)$,

$\left(\mathrm{S}^{*} \mathrm{~A}\right)_{\mathrm{ij}}=$ Interaction between Strain and Age,

$\left(\mathrm{S}^{*} \mathrm{~T}\right)_{\mathrm{ik}}=$ Interaction between Strain and 
Egg type,

$\left(\mathrm{A}^{*} \mathrm{~T}\right)_{\mathrm{jk}}=$ Interaction between Age and Egg type,

$\left(\mathrm{S}^{*} \mathrm{~A} * \mathrm{~T}\right)_{\mathrm{ijk}}=$ Interaction between Strain, Age and Egg type,

$\mathrm{e}_{\mathrm{ijk} \mathrm{l}}=$ Experimental error.

When significant differences between means were found, means were separated using Duncan's multiple range tests.

\section{RESULTS AND DISCUSSION}

\section{Egg weight \& egg weight loss}

Egg weights at 0 and 18 days of incubation as affected by strain, egg type, breeder age and their interactions are showed in Tables (1 and 2). Egg weight was significantly affected by strain, the white layer hens produced significantly heavier egg weight compared to the brown one. The previous results extended also egg weight either at 0 or 18 days of incubation period. Egg weight at 0 day of incubation significantly increased as the layers age progressed. The same trend was noticed by Rizzi and Chiericato (2005); Johnston and Gous (2007) and Rayan (2013) they pointed that the egg weight increased with the hens' age.

Egg weight at 0 and 18 days of incubation was significantly heaviest in large egg type (73 and $64.86 \mathrm{~g}$, respectively) and the lowest value in rough egg type (60.10 and $52.60 \mathrm{~g}$, respectively) (Table 1). Regarding interaction effect, egg weight at 0 day of incubation was significantly affected by interaction between strain and egg type only.

Data of Table (3) indicated that there were no significant differences between strains for relative egg weight loss. Weight loss (\%) was significantly affected by breeder age. The relative egg weight loss increases with advancing of breeder age. Khabisi et al. (2012) reported that egg weight loss during incubation was increased with breeder age.

Egg weight loss (\%) was significantly higher in rough and shape abnormal eggs compared to their counterparts. The minimum percentage of weight loss was recorded in normal eggs (10.72\%). All potential interactions significantly affected weight loss $(\%)$, except those between strain and breeder age ( $\left.S^{*} A\right)$.

\section{Fertility \& hatchability percentages}

Fertility and hatchability (\%) for different egg types, strain and layer breeder ages are summarized in Table (4). The normal, Large and shape abnormality eggs of brown strain had better fertility percentage (92\%); while, rough eggs of brown strain at 46 and 60 wks of breeder age had the lowest fertility percentage (74 and $54 \%$, respectively). Hatchability percentage of fertile eggs for rough eggs of each brown or white strains was the lowest in comparison with chicks from other egg types at different breeder ages. Hatchability percentage decreases with advancing of breeder age for chicks hatched from all studied egg types, the same trend was observed by Abudabos (2010), who stated that hen's age affected hatchability and there was reduction of hatchability with advance age.

\section{Egg and eggshell traits}

Data of Table (5) indicated that W-36 layer hens produced eggs with significantly higher shape index compared to the brown one. The present results revealed that there was a significant decrease in shape index 


\section{Strain - Egg type - Breeder age - Chick quality - Hatching traits.}

with advanced of breeder age. Same trend of egg shape index decrease was reported by Gunlu et al. (2003) and Brand et al. (2004), who pointed that shape index of eggs decreased with age because shape index is directly proportional to egg width and it is inversely related to egg length, which implies that with increasing age, the rate at which eggs becomes longer is faster than rate of being wider. Large egg type was significantly the highest in shape index (79.88 \%); conversely, shape abnormal egg type had the lowest value of shape index compared to other egg types. All potential interactions significantly affected shape index.

Brown strain produced eggs with higher $(\mathrm{P} \leq 0.05)$ shell percentage compared to the white one (Table 6). Similar trend was reported by Scott and Silversides (2000), Renema et al. (2001). Shell percentage significantly decreases with hen's age. Silversides and Scott (2001), Rayan (2013) noticed that shell percentage decreases gradually with hen's ages. Shell percentage was significantly affected by interaction between strain $\times$ egg type $\left(\mathrm{S}^{*} \mathrm{~T}\right)$ and age $\times$ egg type $\left(\mathrm{A}^{*} \mathrm{~T}\right)$.

Data of Table (7) showed that total pores per egg was significantly affected by strain, whereas white eggs recorded significantly higher total pores per egg (7583) compared to the brown one (7249). Total pores significantly increase with breeder age. The increasing in pores number related to advancing of age could be attributed to weight and volume of egg dramatically increased with age. Large egg type was significantly higher in total pores (8165) compared to other egg types. Total pores was significantly affected by interaction between strain $\times$ egg type $\left(\mathrm{S}^{* \mathrm{~T}}\right)$ only.

Tables (8 and 9) clarify that white eggs significantly recorded higher eggshell surface area compared to the brown one. Shell area increased as age increased. Large egg type was significantly higher in eggshell area compared to other egg types.

Concerninig shell index, brown eggs significantly recorded higher shell index compared to the white one. Eggshell index significantly decreases as age increased. This result agrees with those of Rayan et al. (2010). Large and normal egg types were significantly higher in shell index (8.61 and 8.51, respectively); while, rough egg type had the lowest value (8.07) compared to other egg types.

\section{Stages of embryonic mortality}

Data of Table (10) clarify percentages of embryonic mortality stages for different egg types, strain and layer breeder ages. Generally, embryonic mortality had varied between different layer breeder's age. Fairchild et al. (2002) observed the same trend. Kuurman et al. (2003) reported that embryonic mortality in chicken is not uniformly distributed during the incubation period, almost $65 \%$ of embryonic mortality take place in two stages: early (at 4 days), and a late phase (at 19 days) of incubation. Egg with rough shell type was the highest of embryonic mortality percentage in early stage (0-7 d of incubation) for each brown and $\mathrm{W}-36$ strains $(35.1,29.5,55.6$ and 29.3, respectively) at 46 and 60 wks of age. compared to other egg types; followed by eggs with shape abnormality. 


\section{Chick quality}

Day-old chick weights as affected by strain, egg type, breeder age and their interactions are presented in Table (11). Chicks of W36 strain significantly recorded higher body weight compared to the brown one. The heavier chick weight associated with white breeder hens could be attributed to that white breeder hens significantly produced heavier egg weight compared to the brown one. While, Silversides and Scott (2001) and Tona et al. (2002) clarified that egg weight is a dominant factor affecting chick weight at hatch. Chick weight did not significantly affect by age. This result in agreement with those reported by Khabisi et al. (2012) who mentioned that large egg type was significantly higher in chick weight $(46.85 \mathrm{~g})$, followed by abnormal eggs (41.12 g) compared to other egg types. All potential interactions significantly affected chick weight, except interaction between $S^{*}$ A.

Data of Table (12) clarifies Tona score as affected by strain, egg type, breeder age, and their interactions. There were no significant differences between strains for Tona score as indication of chick quality. Tona score decreased as the layer breeder age progressed.
Rough egg type had the lowest value of Tona score $(78.25 \%)$; whereas, there were no significant differences between other studied egg types ((normal (99.80), large (95.65), and abnormal (95.25)).

Tona score was significantly affected by all potential interactions, that means the expression of this trait was different based on strain, layer breeder age and egg type.

\section{FINALLY,}

It could be cncluded that practically we can benefit from large and shape abnormality eggs, because there is no significant difference between them and normal eggs for most eggshell characteristics, chick quality and fertility \& hatchability percentages. Conversely, rough eggshell type. 
Table (1): Egg weight at zero day of incubation as affected by strain, egg type, breeder age and their interactions (Mean \pm SE).

\begin{tabular}{|c|c|c|c|c|c|c|c|c|c|c|c|c|c|}
\hline \multirow[b]{2}{*}{$\begin{array}{l}\text { Strain } \\
(\mathrm{S})\end{array}$} & \multirow[b]{2}{*}{$\begin{array}{l}\text { Age } \\
\text { (A) }\end{array}$} & \multicolumn{4}{|c|}{ Egg type $(\mathrm{T})$} & \multirow[b]{2}{*}{ Overall } & \multicolumn{7}{|c|}{ Prob. } \\
\hline & & Normal & Large & Rough & $\begin{array}{l}\text { Shape } \\
\text { abnormal }\end{array}$ & & $\begin{array}{l}\text { Strain } \\
(\mathrm{S})\end{array}$ & $\begin{array}{l}\text { Age } \\
\text { (A) }\end{array}$ & $\begin{array}{l}\text { Egg } \\
\text { type } \\
\text { (T) }\end{array}$ & $\mathrm{S}^{*} \mathrm{~A}$ & $\mathrm{~S} * \mathrm{~T}$ & $\mathrm{~A}^{* \mathrm{~T}}$ & $\mathrm{~S}^{*} \mathrm{~A} * \mathrm{~T}$ \\
\hline \multicolumn{14}{|c|}{ Egg weight at 0 day of incubation } \\
\hline \multirow{10}{*}{ Brown } & 46 & 59.43 & 71.67 & 56.87 & 59.27 & 62.66 & 0.0001 & 0.001 & 0.0001 & NS & 0.001 & NS & NS \\
\hline & & \pm 0.52 & \pm 0.39 & \pm 0.82 & \pm 0.87 & & & & & & & & \\
\hline & 60 & 62.07 & 71.88 & 58.45 & 61.13 & 64.13 & & & & & & & \\
\hline & & \pm 0.55 & \pm 0.39 & \pm 0.83 & \pm 0.99 & & & & & & & & \\
\hline & Overall & 60.75 & 71.78 & 57.66 & 60.20 & & & & & & & & \\
\hline & 46 & 62.83 & 73.15 & 62.21 & 65.77 & 65.99 & & & & & & & \\
\hline & & \pm 0.50 & \pm 0.45 & \pm 0.93 & \pm 1.02 & & & & & & & & \\
\hline & 60 & 63.40 & 75.30 & 62.86 & 65.35 & 66.73 & & & & & & & \\
\hline & & \pm 0.60 & \pm 0.43 & \pm 0.77 & \pm 0.97 & & & & & & & & \\
\hline & Overall & 63.12 & 74.23 & 62.54 & 65.56 & & & & & & & & \\
\hline \multirow[b]{2}{*}{ Overall } & \multicolumn{2}{|c|}{ Strain } & & & \multicolumn{2}{|c|}{ Breeder age, (wk) } & & & \multicolumn{5}{|c|}{ Egg type } \\
\hline & $\begin{array}{l}\text { Brown } \\
62.60^{\mathrm{b}}\end{array}$ & $\begin{array}{c}\text { W-36 } \\
66.36^{\mathrm{a}}\end{array}$ & & & $\begin{array}{c}46 \\
63.90^{\mathrm{b}}\end{array}$ & $\begin{array}{c}60 \\
65.06^{\mathrm{a}}\end{array}$ & & & $\begin{array}{c}\text { Normal } \\
61.93^{\mathrm{b}}\end{array}$ & $\begin{array}{l}\text { Large } \\
73.00^{\mathrm{a}}\end{array}$ & $\begin{array}{l}\text { Rough } \\
60.10^{\mathrm{c}}\end{array}$ & $\begin{array}{r}a b 1 \\
6 \\
\end{array}$ & $\begin{array}{l}\text { ormal } \\
.88^{b}\end{array}$ \\
\hline
\end{tabular}

${ }^{\mathrm{a} \text { and } \mathrm{b}}$ Means within the same main effects with different letters are significantly differed, NS= Non-significant. 
Table (2): Egg weight at 18 days of incubation as affected by strain, egg type, breeder age and their interactions (Mean \pm SE).

\begin{tabular}{|c|c|c|c|c|c|c|c|c|c|c|c|c|c|}
\hline \multirow[b]{2}{*}{$\begin{array}{l}\text { Strain } \\
(\mathrm{S})\end{array}$} & \multirow[b]{2}{*}{$\begin{array}{l}\text { Age } \\
(\mathrm{A})\end{array}$} & \multicolumn{4}{|c|}{ Egg type $(\mathrm{T})$} & \multirow[b]{2}{*}{ Overall } & \multicolumn{7}{|c|}{ Prob. } \\
\hline & & Normal & Large & Rough & $\begin{array}{l}\text { Shape } \\
\text { abnormal }\end{array}$ & & $\begin{array}{l}\text { Strain } \\
\text { (S) }\end{array}$ & $\begin{array}{l}\text { Age } \\
\text { (A) }\end{array}$ & $\begin{array}{l}\text { Egg } \\
\text { type } \\
(\mathrm{T})\end{array}$ & $\mathrm{S}^{*} \mathrm{~A}$ & $\mathrm{~S}^{*} \mathrm{~T}$ & $\mathrm{~A}^{*} \mathrm{~T}$ & $\mathrm{~S}^{*} \mathrm{~A} * \mathrm{~T}$ \\
\hline \multicolumn{14}{|c|}{ Egg weight at 18 days of incubation } \\
\hline \multirow{5}{*}{ Brov } & 46 & 55.45 & 66.47 & 50.85 & 51.04 & 55.95 & 0.0001 & NS & 0.0001 & 0.01 & 0.0001 & NS & 0.0001 \\
\hline & & \pm 0.53 & \pm 0.54 & \pm 0.93 & \pm 1.03 & & & & & & & & \\
\hline & 60 & 54.37 & 62.58 & 49.42 & 52.73 & 54.78 & & & & & & & \\
\hline & & \pm 0.68 & \pm 0.65 & \pm 1.03 & \pm 1.42 & & & & & & & & \\
\hline & Overall & 54.91 & 64.53 & 50.14 & 51.89 & & & & & & & & \\
\hline \multirow{5}{*}{$\mathrm{W}-36$} & 46 & 55.67 & 65.59 & 56.85 & 61.07 & 59.80 & & & & & & & \\
\hline & & \pm 0.55 & \pm 0.55 & \pm 1.09 & \pm 1.09 & & & & & & & & \\
\hline & 60 & 55.53 & 64.88 & 51.82 & 53.57 & 56.45 & & & & & & & \\
\hline & & \pm 0.65 & \pm 0.68 & \pm 1.00 & \pm 1.09 & & & & & & & & \\
\hline & Overall & 55.60 & 65.24 & 54.34 & 57.32 & & & & & & & & \\
\hline \multirow[b]{2}{*}{ Overall } & \multicolumn{2}{|c|}{ Strain } & & & \multicolumn{2}{|c|}{ Breeder age, (wk) } & & & \multicolumn{5}{|c|}{ Egg type } \\
\hline & $\begin{array}{l}\text { Brown } \\
55.93^{\mathrm{b}}\end{array}$ & $\begin{array}{c}\mathrm{W}-36 \\
58.23^{\mathrm{a}}\end{array}$ & & & $\begin{array}{c}46 \\
57.99\end{array}$ & $\begin{array}{c}60 \\
56.18\end{array}$ & & & $\begin{array}{c}\text { Normal } \\
55.27^{\mathrm{b}}\end{array}$ & $\begin{array}{l}\text { Large } \\
64.86^{\mathrm{a}}\end{array}$ & $\begin{array}{l}\text { Rough } \\
52.60^{c}\end{array}$ & $\begin{array}{r}\mathrm{abn} \\
5\end{array}$ & $\begin{array}{l}\text { ormal } \\
.72^{b}\end{array}$ \\
\hline
\end{tabular}

${ }^{\mathrm{a} \text { and } \mathrm{b}}$ Means within the same main effects with different letters are significantly differed, NS= Non-significant. 
Table (3): Relative egg weight loss (0-18 days) as affected by strain, egg type, breeder age and their interactions (Mean \pm SE).

\begin{tabular}{|c|c|c|c|c|c|c|c|c|c|c|c|c|c|}
\hline \multirow[b]{2}{*}{$\begin{array}{l}\text { Strain } \\
\text { (S) }\end{array}$} & \multirow[b]{2}{*}{$\begin{array}{l}\text { Age } \\
\text { (A) }\end{array}$} & \multicolumn{4}{|c|}{ Egg type (T) } & \multirow[b]{2}{*}{ Overall } & \multicolumn{7}{|c|}{ Prob. } \\
\hline & & Normal & Large & Rough & $\begin{array}{l}\text { Shape } \\
\text { abnormal }\end{array}$ & & $\begin{array}{l}\text { Strain } \\
\text { (S) }\end{array}$ & $\begin{array}{l}\text { Age } \\
\text { (A) }\end{array}$ & $\begin{array}{l}\text { Egg } \\
\text { type } \\
\text { (T) }\end{array}$ & $\mathrm{S}^{*} \mathrm{~A}$ & $\mathrm{~S} * \mathrm{~T}$ & $\mathrm{~A}^{*} \mathrm{~T}$ & S*A*T \\
\hline \multicolumn{14}{|c|}{ Egg weight loss (\%) } \\
\hline \multirow{4}{*}{ Brown } & 46 & $\begin{array}{c}6.27 \\
+0.38\end{array}$ & $\begin{array}{r}7.28 \\
+0.47\end{array}$ & $\begin{array}{r}11.61 \\
+1.04\end{array}$ & $\begin{array}{r}13.93 \\
+1.21\end{array}$ & 9.77 & NS & 0.0001 & 0.0001 & NS & 0.0001 & 0.01 & 0.0001 \\
\hline & 60 & 12.90 & 12.84 & 17.57 & 15.01 & 14.58 & & & & & & & \\
\hline & & \pm 0.68 & \pm 0.73 & \pm 1.14 & \pm 1.22 & & & & & & & & \\
\hline & Overall & 9.59 & 10.06 & 14.59 & 14.47 & & & & & & & & \\
\hline \multirow{5}{*}{$\mathrm{W}-36$} & 46 & 11.39 & 10.32 & 9.52 & 7.23 & 9.62 & & & & & & & \\
\hline & & \pm 0.29 & \pm 0.26 & \pm 0.79 & \pm 0.62 & & & & & & & & \\
\hline & 60 & 12.28 & 13.89 & 18.34 & 18.01 & 15.63 & & & & & & & \\
\hline & & \pm 0.52 & \pm 0.74 & \pm 0.98 & \pm 0.62 & & & & & & & & \\
\hline & Overall & 11.84 & 12.11 & 13.93 & 12.62 & & & & & & & & \\
\hline \multirow[b]{2}{*}{ Overall } & \multicolumn{2}{|c|}{ Strain } & & & \multicolumn{2}{|c|}{ Breeder age, (wk) } & & & \multicolumn{5}{|c|}{ Egg type } \\
\hline & $\begin{array}{c}\text { Brown } \\
11.79\end{array}$ & $\begin{array}{l}\text { W-36 } \\
12.48\end{array}$ & & & $\begin{array}{c}46 \\
9.66^{\mathrm{b}}\end{array}$ & $\begin{array}{c}60 \\
14.84^{\mathrm{a}} \\
\end{array}$ & & & $\begin{array}{c}\text { Normal } \\
10.72^{b}\end{array}$ & $\begin{array}{l}\text { Large } \\
11.10^{\mathrm{b}}\end{array}$ & $\begin{array}{l}\text { Rough } \\
13.96^{\mathrm{a}}\end{array}$ & & $\begin{array}{l}\text { ormal } \\
.31^{\mathrm{a}}\end{array}$ \\
\hline
\end{tabular}

${ }^{a}$ and $b$ Means within the same main effects with different letters are significantly differed, NS= Non-significant. 
Table (4): Fertility and hatchability percentages for different egg types, strains and layer breeder ages.

\begin{tabular}{|c|c|c|c|c|c|}
\hline \multirow{2}{*}{ Egg type } & \multirow{2}{*}{ Strain } & \multicolumn{2}{|c|}{46 wks } & \multicolumn{2}{|c|}{60 wks } \\
\hline & & Fertility (\%) & Hatchability (\%) & Fertility (\%) & Hatchability (\%) \\
\hline \multirow{2}{*}{ Normal eggs } & Brown & 92 & 87 & 90 & 80 \\
\hline & $\mathrm{W}-36$ & 98 & 91.8 & 96 & 81.3 \\
\hline \multirow{2}{*}{ Large eggs } & Brown & 92 & 60.9 & 94 & 48.9 \\
\hline & $\mathrm{W}-36$ & 88 & 50 & 92 & 43.5 \\
\hline \multirow{2}{*}{ Rough shell } & Brown & 74 & 32.4 & 54 & 14.8 \\
\hline & $\mathrm{W}-36$ & 88 & 36.4 & 82 & 24.4 \\
\hline \multirow{2}{*}{ Shape abnormality } & Brown & 92 & 54.3 & 72 & 41.7 \\
\hline & $\mathrm{W}-36$ & 90 & 62.2 & 78 & 33.3 \\
\hline
\end{tabular}

Fertility and hatchability percentages calculated from fertile eggs. 
Table (5): Egg shape index as affected by strain, egg type, breeder age and their interactions (Mean $\pm \mathrm{SE}$ ).

\begin{tabular}{|c|c|c|c|c|c|c|c|c|c|c|c|c|c|}
\hline \multirow[b]{2}{*}{$\begin{array}{l}\text { Strain } \\
(\mathrm{S})\end{array}$} & \multirow[b]{2}{*}{$\begin{array}{l}\text { Age } \\
\text { (A) }\end{array}$} & \multicolumn{4}{|c|}{ Egg type (T) } & \multirow[b]{2}{*}{ Overall } & \multicolumn{7}{|c|}{ Prob. } \\
\hline & & Normal & Large & Rough & $\begin{array}{l}\text { Shape } \\
\text { abnormal }\end{array}$ & & $\begin{array}{l}\text { Strain } \\
(\mathrm{S})\end{array}$ & $\begin{array}{l}\text { Age } \\
(\mathrm{A})\end{array}$ & $\begin{array}{l}\text { Egg } \\
\text { type } \\
\text { (T) }\end{array}$ & $\mathrm{S}^{*} \mathrm{~A}$ & $\mathrm{~S} * \mathrm{~T}$ & $\mathrm{~A} * \mathrm{~T}$ & $\mathrm{~S}^{*} \mathrm{~A}^{*} \mathrm{~T}$ \\
\hline \multicolumn{14}{|c|}{ Egg shape index $(\%)$} \\
\hline \multirow{5}{*}{ Brown } & 46 & 79.92 & 82.32 & 79.11 & 79.52 & 80.22 & 0.001 & 0.0001 & 0.0001 & 0.001 & 0.0001 & 0.001 & 0.05 \\
\hline & & \pm 0.29 & \pm 0.35 & \pm 0.39 & \pm 0.58 & & & & & & & & \\
\hline & 60 & 78.01 & 78.75 & 76.52 & 73.67 & 76.74 & & & & & & & \\
\hline & & \pm 0.36 & \pm 0.50 & \pm 0.37 & \pm 0.55 & & & & & & & & \\
\hline & Overall & 78.97 & 80.54 & 77.82 & 76.60 & & & & & & & & \\
\hline \multirow{5}{*}{$\mathrm{W}-36$} & 46 & 79.30 & 80.46 & 81.00 & 80.49 & 80.31 & & & & & & & \\
\hline & & \pm 0.27 & \pm 0.38 & \pm 0.42 & \pm 0.51 & & & & & & & & \\
\hline & 60 & 77.64 & 77.99 & 78.83 & 78.10 & 78.14 & & & & & & & \\
\hline & & \pm 0.40 & \pm 0.46 & \pm 0.48 & \pm 0.79 & & & & & & & & \\
\hline & Overall & 78.47 & 79.23 & 79.92 & 79.30 & & & & & & & & \\
\hline \multirow{3}{*}{ Overall } & \multicolumn{2}{|c|}{ Strain } & & & \multicolumn{2}{|c|}{ Breeder age, (wk) } & & & \multicolumn{5}{|c|}{ Egg type } \\
\hline & Brown & $\mathrm{W}-36$ & & & 46 & 60 & & & Normal & Large & Rough & abn & ormal \\
\hline & $78.48^{\mathrm{b}}$ & $79.23^{\mathrm{a}}$ & & & $80.26^{\mathrm{a}}$ & $77.44^{\mathrm{b}}$ & & & $78.72^{b}$ & $79.88^{\mathrm{a}}$ & $78.87^{\mathrm{b}}$ & & $.94^{\mathrm{c}}$ \\
\hline
\end{tabular}

${ }^{\mathrm{a} \text { and } b}$ Means within the same main effects with different letters are significantly differed, NS= Non-significant. 
Table (6): Shell percentage as affected by strain, egg type, breeder age and their interactions (Mean \pm SE).

\begin{tabular}{|c|c|c|c|c|c|c|c|c|c|c|c|c|c|}
\hline \multirow[b]{2}{*}{$\begin{array}{l}\text { Strain } \\
(\mathrm{S})\end{array}$} & \multirow[b]{2}{*}{$\begin{array}{l}\text { Age } \\
\text { (A) }\end{array}$} & \multicolumn{4}{|c|}{ Egg type (T) } & \multirow[b]{2}{*}{ Overall } & \multicolumn{7}{|c|}{ Prob. } \\
\hline & & Normal & Large & Rough & $\begin{array}{l}\text { Shape } \\
\text { abnormal }\end{array}$ & & $\begin{array}{l}\text { Strain } \\
(\mathrm{S})\end{array}$ & $\begin{array}{l}\text { Age } \\
\text { (A) }\end{array}$ & \begin{tabular}{|l} 
Egg \\
type \\
$(\mathrm{T})$
\end{tabular} & $S^{*} A$ & $\mathrm{~S}^{* \mathrm{~T}}$ & $\mathrm{~A} * \mathrm{~T}$ & $\mathrm{~S} * \mathrm{~A} * \mathrm{~T}$ \\
\hline \multicolumn{14}{|c|}{ Shell percentage } \\
\hline \multirow{3}{*}{ Brown } & 46 & $\begin{array}{l}10.51 \\
\pm 0.18\end{array}$ & $\begin{array}{c}9.41 \\
\pm 0.17\end{array}$ & $\begin{array}{l}10.17 \\
\pm 0.35\end{array}$ & $\begin{array}{c}9.61 \\
\pm 0.30\end{array}$ & 9.92 & 0.0001 & 0.0003 & 0.0001 & NS & 0.05 & 0.0003 & NS \\
\hline & 60 & $\begin{array}{c}9.82 \\
\pm 0.19\end{array}$ & $\begin{array}{c}8.57 \\
\pm 0.30\end{array}$ & $\begin{array}{c}9.10 \\
\pm 0.99\end{array}$ & $\begin{array}{l}10.92 \\
\pm 0.28\end{array}$ & 9.60 & & & & & & & \\
\hline & Overall & 10.16 & 8.99 & 9.63 & 10.26 & & & & & & & & \\
\hline \multirow{4}{*}{$\mathrm{W}-36$} & 46 & $\begin{array}{c}9.51 \\
\pm 0.16\end{array}$ & $\begin{array}{c}8.99 \\
\pm 0.21\end{array}$ & $\begin{array}{c}9.08 \\
\pm 0.26\end{array}$ & $\begin{array}{c}8.78 \\
\pm 0.28\end{array}$ & 9.09 & & & & & & & \\
\hline & 60 & 8.33 & 7.90 & 8.36 & 8.54 & 8.28 & & & & & & & \\
\hline & & \pm 0.21 & \pm 0.29 & \pm 0.48 & \pm 0.47 & & & & & & & & \\
\hline & Overall & 8.92 & 8.44 & 8.72 & 8.66 & & & & & & & & \\
\hline \multirow[b]{2}{*}{ Overall } & \multicolumn{2}{|c|}{ Strain } & & & \multicolumn{2}{|c|}{ Breeder age, (wk) } & & & \multicolumn{5}{|c|}{ Egg type } \\
\hline & $\begin{array}{c}\text { Brown } \\
9.84^{\mathrm{a}}\end{array}$ & $\begin{array}{l}\text { W-36 } \\
8.81^{\mathrm{b}}\end{array}$ & & & $\begin{array}{c}46 \\
9.56^{\mathrm{a}}\end{array}$ & $\begin{array}{c}60 \\
8.95^{\mathrm{b}}\end{array}$ & & & $\begin{array}{c}\text { Normal } \\
9.58^{\mathrm{a}}\end{array}$ & $\begin{array}{l}\text { Large } \\
8.80^{\mathrm{b}}\end{array}$ & $\begin{array}{c}\text { Rough } \\
9.22^{\mathrm{a}}\end{array}$ & $\begin{array}{r}\text { Shape a } \\
9 .\end{array}$ & $\begin{array}{l}\text { bnormal } \\
9^{\text {a }}\end{array}$ \\
\hline
\end{tabular}

${ }^{\mathrm{a} \text { and } \mathrm{b}}$ Means within the same main effects with different letters are significantly differed, NS= Non-significant. 
Table (7): Total pores per egg as affected by strain, egg type, breeder age and their interactions (Mean \pm SE).

\begin{tabular}{|c|c|c|c|c|c|c|c|c|c|c|c|c|c|}
\hline \multirow[b]{2}{*}{$\begin{array}{l}\text { Strain } \\
(\mathrm{S})\end{array}$} & \multirow[b]{2}{*}{$\begin{array}{l}\text { Age } \\
\text { (A) }\end{array}$} & \multicolumn{4}{|c|}{ Egg type (T) } & \multirow[b]{2}{*}{ Overall } & \multicolumn{7}{|c|}{ Prob. } \\
\hline & & Normal & Large & Rough & $\begin{array}{l}\text { Shape } \\
\text { abnormal }\end{array}$ & & $\begin{array}{l}\text { Strain } \\
(\mathrm{S})\end{array}$ & $\begin{array}{r}\text { Age } \\
\text { (A) }\end{array}$ & $\begin{array}{l}\text { Egg } \\
\text { type } \\
\text { (T) }\end{array}$ & $\mathrm{S}^{*} \mathrm{~A}$ & $\mathrm{~S} * \mathrm{~T}$ & $\mathrm{~A} * \mathrm{~T}$ & $\mathrm{~S}^{*} \mathrm{~A} * \mathrm{~T}$ \\
\hline \multicolumn{14}{|c|}{ Total pores per egg } \\
\hline \multirow{6}{*}{ Brown } & 46 & 6973 & 8051 & 6737 & 6954 & 7178 & 0.0001 & 0.001 & 0.0001 & NS & 0.002 & NS & NS \\
\hline & & \pm 46.4 & \pm 33.7 & \pm 74.7 & \pm 78.8 & & & & & & & & \\
\hline & 60 & 7209 & 8069 & 6880 & 7119 & 7319 & & & & & & & \\
\hline & & \pm 49.0 & \pm 33.9 & \pm 75.4 & \pm 88.3 & & & & & & & & \\
\hline & Overall & 7091 & 8060 & 6808 & 7036 & & & & & & & & \\
\hline & 46 & 7277 & 8178 & 7217 & 7531 & 7550 & & & & & & & \\
\hline \multirow{4}{*}{$\mathrm{W}-36$} & & \pm 44.6 & \pm 38.8 & \pm 83.2 & \pm 91.4 & & & & & & & & \\
\hline & 60 & 7327 & 8362 & 7277 & 7494 & 7615 & & & & & & & \\
\hline & & \pm 53.4 & \pm 36.1 & \pm 67.8 & \pm 86.0 & & & & & & & & \\
\hline & Overall & 7302 & 8270 & 7247 & 7512 & & & & & & & & \\
\hline \multirow[b]{2}{*}{ Overall } & \multicolumn{2}{|c|}{ Strain } & & & \multicolumn{2}{|c|}{ Breeder age, (wk) } & & & \multicolumn{5}{|c|}{ Egg type } \\
\hline & $\begin{array}{c}\text { Brown } \\
7249^{b}\end{array}$ & $\begin{array}{l}\mathrm{W}-36 \\
7583^{\mathrm{a}}\end{array}$ & & & $\begin{array}{c}46 \\
7365^{\mathrm{b}}\end{array}$ & $\begin{array}{c}60 \\
7467^{\mathrm{a}}\end{array}$ & & & $\begin{array}{c}\text { Normal } \\
7196^{\mathrm{b}}\end{array}$ & $\begin{array}{l}\text { Large } \\
8165^{\mathrm{a}}\end{array}$ & $\begin{array}{l}\text { Rough } \\
7028^{c}\end{array}$ & $\overline{a b}$ & $\begin{array}{l}\text { ormal } \\
274^{b}\end{array}$ \\
\hline
\end{tabular}

${ }^{a}$ and $b$ Means within the same main effects with different letters are significantly differed, NS= Non-significant. 
Table (8): Eggshell surface area as affected by strain, egg type, breeder age and their interactions (Mean \pm SE).

\begin{tabular}{|c|c|c|c|c|c|c|c|c|c|c|c|c|c|}
\hline \multirow[b]{2}{*}{$\begin{array}{l}\text { Strain } \\
(\mathrm{S})\end{array}$} & \multirow[b]{2}{*}{$\begin{array}{l}\text { Age } \\
(\mathrm{A})\end{array}$} & \multicolumn{4}{|c|}{ Egg type $(\mathrm{T})$} & \multirow[b]{2}{*}{ Overall } & \multicolumn{7}{|c|}{ Prob. } \\
\hline & & Normal & Large & Rough & $\begin{array}{l}\text { Shape } \\
\text { abnormal }\end{array}$ & & $\begin{array}{l}\text { Strain } \\
(\mathrm{S})\end{array}$ & $\begin{array}{l}\text { Age } \\
(\mathrm{A})\end{array}$ & $\begin{array}{l}\text { Egg } \\
\text { type } \\
\text { (T) }\end{array}$ & $\mathrm{S}^{*} \mathrm{~A}$ & $\mathrm{~S} * \mathrm{~T}$ & $\mathrm{~A} * \mathrm{~T}$ & $\mathrm{~S} * \mathrm{~A} * \mathrm{~T}$ \\
\hline \multicolumn{14}{|c|}{ Eggshell surface area $\left(\mathrm{mg} / \mathrm{cm}^{2}\right)$} \\
\hline \multirow{5}{*}{ Brown } & 46 & 68.81 & 74.62 & 69.07 & 68.95 & 70.36 & 0.0001 & 0.02 & 0.0001 & $\mathrm{NS}$ & 0.01 & NS & 0.001 \\
\hline & & \pm 0.24 & \pm 0.22 & \pm 0.33 & \pm 0.46 & & & & & & & & \\
\hline & 60 & 68.79 & 74.08 & 69.05 & 70.63 & 70.64 & & & & & & & \\
\hline & & \pm 0.29 & \pm 0.25 & \pm 0.41 & \pm 0.48 & & & & & & & & \\
\hline & Overall & 68.8 & 74.35 & 69.06 & 69.79 & & & & & & & & \\
\hline \multirow{5}{*}{$\mathrm{W}-36$} & 46 & 69.33 & 74.25 & 70.75 & 72.09 & 71.60 & & & & & & & \\
\hline & & \pm 0.27 & \pm 0.21 & \pm 0.42 & \pm 0.46 & & & & & & & & \\
\hline & 60 & 69.96 & 75.89 & 70.87 & 71.95 & 72.17 & & & & & & & \\
\hline & & \pm 0.32 & \pm 0.26 & \pm 0.35 & \pm 0.47 & & & & & & & & \\
\hline & Overall & 69.64 & 75.07 & 70.81 & 72.02 & & & & & & & & \\
\hline \multirow{3}{*}{ Overall } & \multicolumn{2}{|c|}{ Strain } & & & \multicolumn{2}{|c|}{ Breeder age, (wk) } & & & \multicolumn{5}{|c|}{ Egg type } \\
\hline & Brown & W-36 & & & 46 & 60 & & & Normal & Large & Rough & Shape & onormal \\
\hline & $70.50^{\mathrm{b}}$ & $71.89^{\mathrm{a}}$ & & & $70.98^{b}$ & $71.40^{\mathrm{a}}$ & & & $69.22^{\mathrm{d}}$ & $74.71^{\mathrm{a}}$ & $69.93^{c}$ & & $90^{\mathrm{b}}$ \\
\hline
\end{tabular}

and b Means within the same main effects with different letters are significantly differed, NS= Non-significant. 
Table (9): Shell index as affected by strain, egg type, breeder age and their interactions (Mean \pm SE).

\begin{tabular}{|c|c|c|c|c|c|c|c|c|c|c|c|c|c|}
\hline \multirow[b]{2}{*}{$\begin{array}{l}\text { Strain } \\
\text { (S) }\end{array}$} & \multirow[b]{2}{*}{$\begin{array}{l}\text { Age } \\
\text { (A) }\end{array}$} & \multicolumn{4}{|c|}{ Egg type (T) } & \multirow[b]{2}{*}{ Overall } & \multicolumn{7}{|c|}{ Prob. } \\
\hline & & Normal & Large & Rough & $\begin{array}{l}\text { Shape } \\
\text { abnormal }\end{array}$ & & $\begin{array}{l}\text { Strain } \\
(\mathrm{S})\end{array}$ & $\begin{array}{r}\text { Age } \\
\text { (A) }\end{array}$ & $\begin{array}{l}\text { Egg } \\
\text { type } \\
\text { (T) }\end{array}$ & $\mathrm{S} * \mathrm{~A}$ & $\mathrm{~S} * \mathrm{~T}$ & A*T & $\mathrm{S}^{*} \mathrm{~A}^{*} \mathrm{~T}$ \\
\hline \multicolumn{14}{|c|}{ Shell index $\left(\mathrm{g} / 100 \mathrm{~cm}^{2}\right)$} \\
\hline \multirow{5}{*}{ Brown } & 46 & 9.00 & 9.06 & 8.57 & 8.16 & 8.70 & 0.0001 & 0.002 & 0.05 & 0.01 & NS & 0.0001 & 0.05 \\
\hline & & \pm 0.16 & \pm 0.16 & \pm 0.29 & \pm 0.25 & & & & & & & & \\
\hline & 60 & 8.88 & 8.35 & 7.69 & 9.67 & 8.65 & & & & & & & \\
\hline & & \pm 0.16 & \pm 0.31 & \pm 0.95 & \pm 0.21 & & & & & & & & \\
\hline & Overall & 8.94 & 8.70 & 8.13 & 8.91 & & & & & & & & \\
\hline \multirow{5}{*}{$\mathrm{W}-36$} & 46 & 8.56 & 8.91 & 8.09 & 8.06 & 8.40 & & & & & & & \\
\hline & & \pm 0.14 & \pm 0.22 & \pm 0.19 & \pm 0.18 & & & & & & & & \\
\hline & 60 & 7.48 & 7.81 & 7.58 & 7.71 & 7.64 & & & & & & & \\
\hline & & \pm 0.17 & \pm 0.28 & \pm 0.44 & \pm 0.32 & & & & & & & & \\
\hline & Overall & 8.02 & 8.36 & 7.83 & 7.88 & & & & & & & & \\
\hline \multirow[b]{2}{*}{ Overall } & \multicolumn{2}{|c|}{ Strain } & & & \multicolumn{2}{|c|}{ Breeder age, (wk) } & & & \multicolumn{5}{|c|}{ Egg type } \\
\hline & $\begin{array}{c}\text { Brown } \\
8.79^{\mathrm{a}}\end{array}$ & $\begin{array}{l}\text { W-36 } \\
8.11^{\mathrm{b}}\end{array}$ & & & $\begin{array}{c}46 \\
8.60^{\mathrm{a}}\end{array}$ & $\begin{array}{c}60 \\
8.21^{\mathrm{b}}\end{array}$ & & & $\begin{array}{c}\text { Normal } \\
8.51^{\mathrm{a}}\end{array}$ & $\begin{array}{l}\text { Large } \\
8.61^{\mathrm{a}}\end{array}$ & $\begin{array}{c}\text { Rough } \\
8.07^{\mathrm{b}}\end{array}$ & $\begin{array}{r}\text { Shape } \\
8 .\end{array}$ & $\begin{array}{l}\text { onormal } \\
2^{\text {ab }}\end{array}$ \\
\hline
\end{tabular}

${ }^{\mathrm{a} \text { and } b}$ Means within the same main effects with different letters are significantly differed, NS= Non-significant. 
Table (10): Percentages of embryonic mortality stages for different egg types, strain and layer breeder ages.

ล

\begin{tabular}{|c|c|c|c|c|c|c|c|c|c|}
\hline \multirow{3}{*}{ Egg type } & \multirow{3}{*}{ Strain } & \multicolumn{8}{|c|}{ Mortality stage } \\
\hline & & \multicolumn{4}{|c|}{$46 \mathrm{wks}$} & \multicolumn{4}{|c|}{60 wks } \\
\hline & & Early & Mid & Late & Pipped & Early & Mid & Late & Pipped \\
\hline \multirow{2}{*}{ Normal eggs } & Brown & 2.2 & 6.5 & 2.2 & 2.2 & 2.2 & 6.7 & 8.9 & 0 \\
\hline & W-36 & 0 & 0 & 8.2 & 0 & 6.3 & 4.1 & 6.3 & 0 \\
\hline \multirow{2}{*}{ Large eggs } & Brown & 6.5 & 8.7 & 8.7 & 13 & 17 & 6.4 & 23.4 & 0 \\
\hline & W-36 & 13.6 & 11.4 & 15.9 & 9.1 & 15.2 & 10.9 & 19.6 & 2.2 \\
\hline \multirow[t]{2}{*}{ Rough shell } & Brown & 35.1 & 13.5 & 13.5 & 0 & 55.6 & 18.5 & 11.1 & 0 \\
\hline & $\mathrm{W}-36$ & 29.5 & 18.2 & 9.1 & 2.3 & 29.3 & 19.5 & 24.4 & 0 \\
\hline \multirow{2}{*}{ Shape abnormality } & Brown & 15.2 & 10.9 & 8.7 & 2.2 & 30.6 & 13.9 & 8.3 & 0 \\
\hline & W-36 & 11.1 & 8.9 & 11.1 & 2.2 & 28.2 & 17.9 & 7.7 & 2.6 \\
\hline
\end{tabular}

Early stage ( 1 to $7 \mathrm{~d}$ ), Mid stage ( 8 to $14 \mathrm{~d}$ ), and Late stage (15 to $21 \mathrm{~d}$ ). 
Table (11): Day-old chick weight as affected by strain, egg type, breeder age and their interactions (Mean $\pm \mathrm{SE})$.

\begin{tabular}{|c|c|c|c|c|c|c|c|c|c|c|c|c|c|}
\hline \multirow[b]{2}{*}{$\begin{array}{l}\text { Strain } \\
\text { (S) }\end{array}$} & \multirow[b]{2}{*}{$\begin{array}{l}\text { Age } \\
\text { (A) }\end{array}$} & \multicolumn{4}{|c|}{ Egg type (T) } & \multirow[b]{2}{*}{ Overall } & \multicolumn{7}{|c|}{ Prob. } \\
\hline & & Normal & Large & Rough & $\begin{array}{l}\text { Shape } \\
\text { abnormal }\end{array}$ & & $\begin{array}{l}\text { Strain } \\
(\mathrm{S})\end{array}$ & $\begin{array}{l}\text { Age } \\
(\mathrm{A})\end{array}$ & $\begin{array}{l}\text { Egg } \\
\text { type } \\
\text { (T) }\end{array}$ & $\mathrm{S}^{*} \mathrm{~A}$ & $\mathrm{~S}^{*} \mathrm{~T}$ & A*T & $\mathrm{S}^{*} \mathrm{~A} * \mathrm{~T}$ \\
\hline \multicolumn{14}{|c|}{ Chick weight (g) } \\
\hline \multirow{6}{*}{ Brown } & 46 & 37.83 & 46.66 & 37.72 & 35.19 & 39.35 & 0.0001 & NS & 0.0001 & NS & 0.0001 & 0.01 & 0.004 \\
\hline & & \pm 0.43 & \pm 0.60 & \pm 1.04 & \pm 0.80 & & & & & & & & \\
\hline & 60 & 38.62 & 44.14 & 34.59 & 39.80 & 39.29 & & & & & & & \\
\hline & & \pm 0.60 & \pm 0.62 & \pm 0.91 & \pm 0.82 & & & & & & & & \\
\hline & Overall & 38.22 & 45.40 & 36.15 & 37.49 & & & & & & & & \\
\hline & 46 & 40.74 & 48.80 & 42.45 & 45.82 & 44.45 & & & & & & & \\
\hline & & \pm 0.54 & \pm 0.55 & \pm 1.48 & \pm 0.96 & & & & & & & & \\
\hline W-36 & 60 & 40.38 & 47.97 & 39.61 & 43.94 & 42.97 & & & & & & & \\
\hline & & \pm 0.48 & \pm 0.70 & \pm 0.94 & \pm 1.55 & & & & & & & & \\
\hline & Overall & 40.56 & 48.38 & 41.03 & 44.88 & & & & & & & & \\
\hline \multirow[b]{2}{*}{ Overall } & \multicolumn{2}{|c|}{ Strain } & & & \multicolumn{2}{|c|}{ Breeder age, (wk) } & & & \multicolumn{5}{|c|}{ Egg type } \\
\hline & $\begin{array}{l}\text { Brown } \\
39.84^{\text {b }}\end{array}$ & $\begin{array}{l}\text { W-36 } \\
43.43^{\mathrm{a}}\end{array}$ & & & $\begin{array}{c}46 \\
41.79\end{array}$ & $\begin{array}{c}60 \\
41.51\end{array}$ & & & $\begin{array}{c}\text { Normal } \\
39.43^{c}\end{array}$ & $\begin{array}{l}\text { Large } \\
46.85^{\mathrm{a}}\end{array}$ & $\begin{array}{l}\text { Rough } \\
39.67^{c}\end{array}$ & $\begin{array}{r}a b \\
4\end{array}$ & $\begin{array}{l}\text { ormal } \\
.12^{\mathrm{b}}\end{array}$ \\
\hline
\end{tabular}

$\mathrm{a}$ and $\mathrm{b}$ Means within the same main effects with different letters are significantly differed, NS= Non-significant. 
Table (12): Tona score as affected by strain, egg type, breeder age and their interactions (Mean \pm SE).

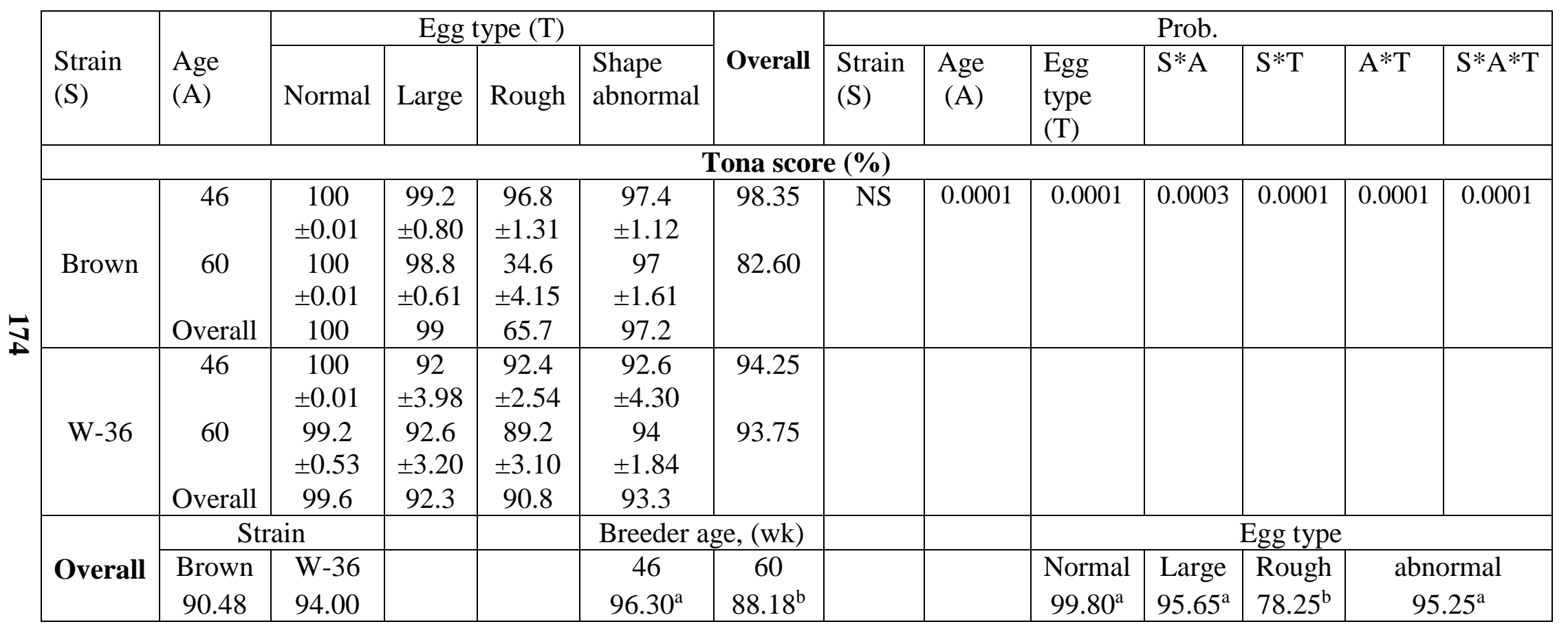

${ }^{\mathrm{a} \text { and } \mathrm{b}}$ Means within the sam main effects with different letters are significantly differed, NS= Non-significant 
Strain - Egg type - Breeder age - Chick quality - Hatching traits.

\section{REFERENCES}

Abudabos, A. 2010. The effect of broiler breeder strain and parent flock age on hatchability and fertile hatchability. Int. J. Poult. Sci., 9 (3): 231-235.

Brand, H.V.D., Parameter H.K. and Kemp B. 2004. Effects of housing system cout door vs cages and age of laying hens on egg characteristics. Br. Poult Sci., 45: 745-752.

Decuypere, E. and Bruggeman, V. 2007. The endocrineinterface of environmental and egg factors affecting chick quality. Poult. Sci., 86: 1037-1042.

Fairchild, B.D., Christensen, V.L., Grimes, J.L., Wineland, M.J. and Bagley, L.G. 2002. Hen age relationship with embryonic mortality and fertility in commercial turkeys. J. of Appl. Poult. Res., 11: 260-265.

Fathi, M.M. and El-Sahar E.A. 1996. Determining the strength of eggshell by using an appropriate apparatus and an equation to calculate egg surface depending on its dimensions. Egypt. Poult. Sci., 16: 285-303.

Gunlu, A., Kiriki, K., Cetin, O. and Carip, M. 2003. Some external and internal quality characteristics of patridge (A. graeca) eggs. Food Agri. Environ., 1: 197-199.

Hamidu, J.A., Fasenko, G.M., Feeddes, J.J.R., O'dea, E.E., Ouellette, C.A.2007. The effect of broiler breeder genetic strain and parent flock age on eggshell conductance and embryonic metabolism. Poult. Sci. 86: 2420-2432.
Heier, B.T. and Jarp, J. 2001. An epidemiological study of the hatchability in broiler breeder flocks. Poult. Sci., 80:1132-1138.

Johnston, S.A. and Gous, R.M. 2007. Modelling the changes in the proportions of the egg components during a laying cycle. Br. Poult. Sci., 48: 347-353.

Khabisi, M.M.; Salahi, A. and Mousavi, S.N. 2012. The influence of egg shell crack types on hatchability and chick quality. Turkish J. Vet. and Anim. Sci., 36: 289-295.

Kuurman, W.W., Bailey, B.A., Koops, W. J. and Grossman, M. 2003. A Model for failure of a chicken embryo to survive incubation. Poult. Sci., 82: 214-222.

Lourens, A., Molenaar, R., Van den Brand, H.E., Heetkamp, M.J.W., Meijerhof, R., Kemp, A. 2006. Effect of egg size on heat production and the transition of energy from egg to hatchling. Poult. Sci. 85: 770-776.

Narushin, V. G., and M. N. Romanov. 2002. Egg physical characteristics and hatchability. World's Poult. Sci. J. 58:297-303.

Peebles, E.D.; Doyle, S.M.; Zumwalt, C.D.; Gerard, P.D.; Latour, M.A.; Boyle, C.R. and Smith, T.W. 2001. Breeder age influences embryogenesis in broiler hatching eggs. Poult. Sci., 80: 272-277.

Rahn, H. and Paganelli, C.V. 1990. Gas fluxes in avian eggs: Driving forces and the pathway for exchange. Comparative Biochemistry and Physiology, 95: 1-15. 
Rayan, G.N. 2013. Genetic differences of productive performance and immuncompetence in two commercial layer strain of chickens. Ph.D. Thesis, Ain Shams University. Cairo, Egypt.

Rayan, G.N., Galal, A., Fathi, M.M. and El-Attar, A.H. 2010. Impact of layer breeder flock age and strain on mechanical and ultrastructural properties of eggshell in chicken. Int. J. Poult. Sci. 9:139-147.

Renema, R.A., Robinson, F.E., Feddes, J.J.R., Fasenko, G.M., and Zuidhof, M.J. 2001. Effects of light intensity from photo photostimulation in four strains of commercial egg layers: 2. Egg production parameters. Poult. Sci. 80:1121-1131.

Rizzi, C., and Chiericato, G.M. 2005. Organic farming production. Effect of age on the productive yield and egg quality of hens of two commercial hybrid lines and two local breeds. Ital. J. Anim. Sci., 4: 160-162.

SAS institute 2002. SAS/STAT User's Guide statistics Ver. 9.1; SAS institute Inc., Cary, NC.

Sauveur, B. 1988. Reproduction des volailles et production d'aufs (Paris, INRA Editions).

Scott, T.A. and Silversides F.G. 2000. Effect of storage and strain of hen on egg quality. Poult. Sci. 79: 1725 - 1729.

Silversides, F.G. and Scott, T.A. 2001. Effect of storage and layer age on quality of eggs from two lines of hens. Poult. Sci., 80: 1240-1245.
Tona, K., Bamelis, F.; Coucke, W. ; Bruggeman, V. and Decuypere, E. 2001. Relationship between broiler breeder's age and egg weight loss and embryonic mortality during incubation in large-scale conditions. J. Appl. Poult. Res., 10: 221-227.

Tona, K., Bamelis, F., De Ketelaere, B., Bruggeman, V., and Decuypere, E. 2002. Effect of induced molting on albumen quality, hatchability, and chick body weight from broiler breeders. Poult. Sci. 81:327-332.

Tona, K., Bamelis, F., De Ketelaere, B., Bruggeman, V., Moraes, V.M.B., Buyse, J., Onagbesan, O., and Decuypere, E. 2003. Effects of egg storage time on spread of hatch, chick quality and chick juvenile growth. Poult. Sci., 82: 736-741.

Tona, K., Bruggeman, V., Onagbesana, O., Bamelis, F., Gbeasor, M., Mertens, $K$ and Decuypere, E. 2005. Dayold chick quality: Relationship to hatching egg quality, adequate incubation practice and prediction of broiler performance. Avian Poult. Biolo. Rev., 16: 109-119.

Wilson, H.R. 1997. Effects of maternal nutrition on hatchability. Poult. Sci., 76: 134-143. 
Strain - Egg type - Breeder age - Chick quality - Hatching traits.

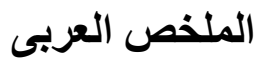

تأثير السلالة، نوع البيض، وعمر أمهات الدجاج والتداخل بينهما على صفات التفريخ وجودة الكتاكيت الناتجة والتان

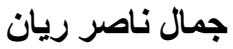

قسم إنتاج الدو اجن، كلية الزراعة - جامعة عين شمس، القاهرة - مصر

تم الحصول على عدد 800 بيضة تفريخ من سلالتي هاى لاين (400 بنى، 400 أبيض) وذلك في أعمار مختلفة

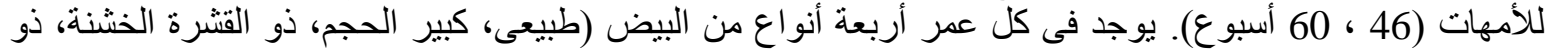

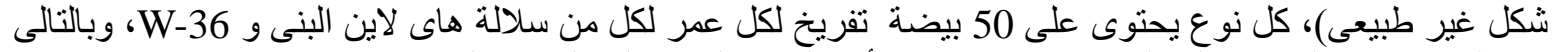

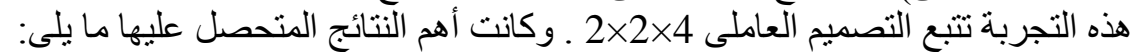

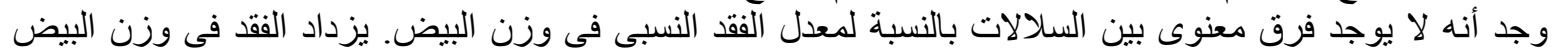

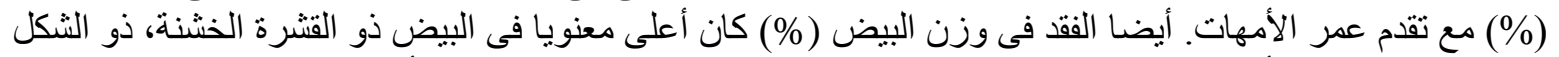

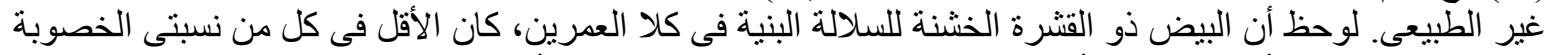

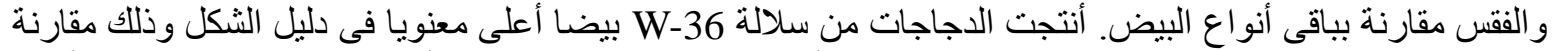

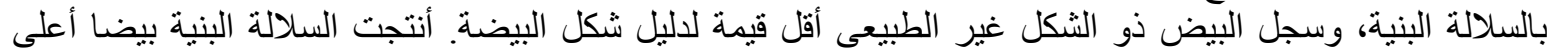

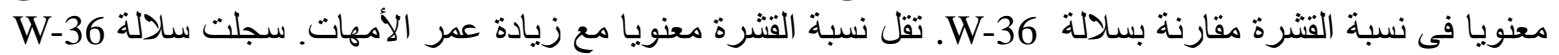

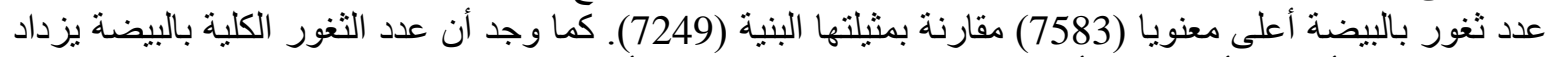

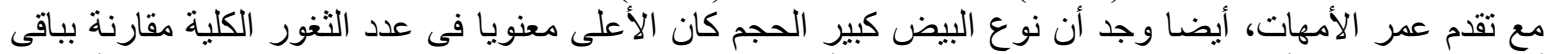

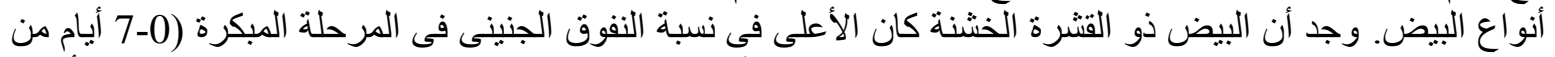

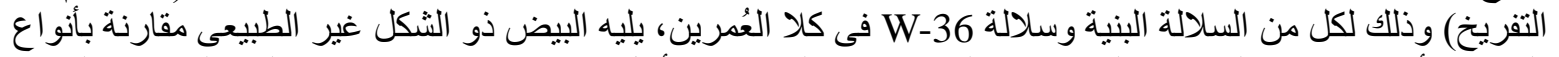

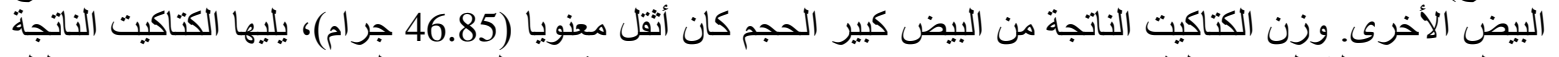

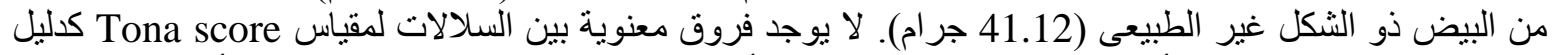

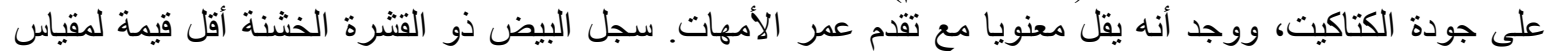
Tona score

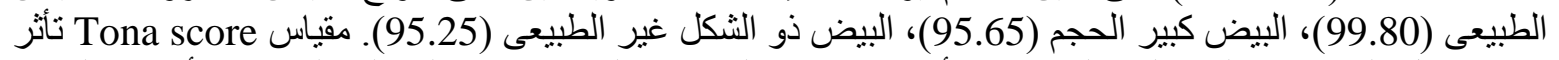

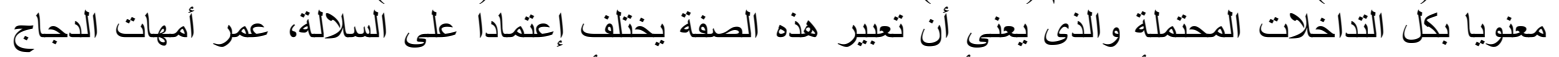

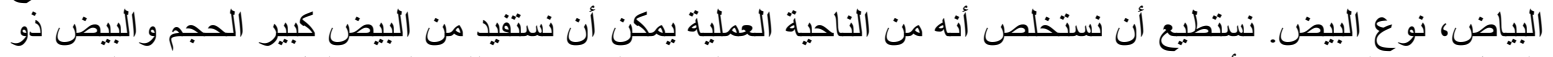

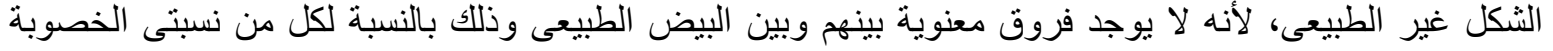

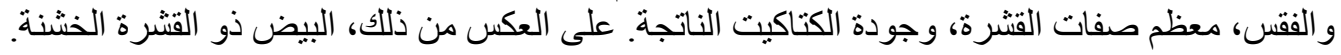

